\title{
Effect of Actomyosin on Heat-induced Gelation of Myosin
}

\author{
Tsutomu YasuI, Makoto Ishioroshi and Kunihiko SAMEJIMA* \\ Department of Animal Science, Faculty of Agriculture, Hokkaido University, \\ Sapporo, Hokkaido 060, Japan \\ * Department of Dairy Science, The College of Dairying, \\ Ebetsu, Hokkaido 069-01, Japan
}

Received November 30, 1981

\begin{abstract}
Myosin and reconstituted actomyosin free of regulatory proteins were mixed in $0.6 \mathrm{M} \mathrm{KCl}$ and $20 \mathrm{~mm}$ phosphate buffer at $\mathrm{pH} 6.0$ and were tested quantitatively for thermally induced gelation properties by measuring the rigidity of the system at $65^{\circ} \mathrm{C}$. Full enhancement of gelation was attained when the weight ratio of myosin-to-actomyosin was about 4 . The addition of regulatory proteins to actomyosin could restore calcium sensitivity of the contractile system, but did not affect the heat-induced gelation of myosin in the presence of actomyosin, suggesting that regulatory proteins play no role in the heat-induced gelation of the system.

Neither the single and double headed subfragments, both capable of interacting with F-actin, nor the helical tail subfragments, devoid of the intracting site with F-actin, exhibited changes in thermogelling properties when mixed with F-actin. However, upon addition to F-actomyosin, the tail subfragments revealed a significant effect on the gelation of actomyosin, whereas the headed subfragments exerted no influence over gelation of the system. These results indicate that the enhancing effect of F-actin on the heat-induced gelation of myosin was brought about solely by the limited amount of F-actomyosin formed in the system, which acts as a cross-linker between the tail portion of bound and free myosin molecules.
\end{abstract}

The manufacture of comminuted processed meat depends on the formation of a functional protein matrix in the product. The products characteristic texture and bite are provided by the properties of this matrix and those parameters can be described in terms of emulsification and/or binding and gelation performance. In our laboratory, a number of studies $^{1 \sim 10)}$ have been completed with purified proteins in an effort to determine which of the myofibrillar proteins are the most essential for the development of gelation properties. The conclusion derived from those results is that gelation in comminuted meat products is related to the physico-chemical properties of myosin and/or actomyosin. Myosin and other components of the myofibrillar protein complex show an intimate relationship to the binding quality of the comminuted meat products, with the greatest contribution coming from myo$\sin ^{1 \sim 4,12 \sim 19)}$ Nakayama and Sato ${ }^{14,15)}$ re- ported that actin and native tropomyosin (a complex of tropomyosin and troponins), as well as myosin were important for the development of viscoeleastic properties of heatset gels of minced vertebrate muscles.

Very recently, Yasui et al., ${ }^{7)}$ Ishioroshi et al., ${ }^{8)}$ who worked with reconstituted actomyosin and Samejima et al. ${ }^{11)}$ who worked with natural actomyosin have carried out comprehensive studies on the heat-induced gelation of myosin in the presence of actin. They observed a remarkable enhancing effect of $\mathrm{F}$ actin on the heat-induced gel formability as measured by the rigidity of myosin in $0.6 \mathrm{M}$ $\mathrm{KCl}$ at $\mathrm{pH} 6.0$ and $65^{\circ} \mathrm{C}$. The effect appeared optimal when the myosin/actin mole ratio in the system came close to 2.7 where $15 \sim 20 \%$ of the total protein existed as actomyosin complex and most of the surplus myosin was found to be free. Those results indicated that the formation of a suitable but relatively small amount of F-actomyosin complex, which acts 
as a cross-linker with free myosin on heating, is a prerequisite for the development of the actin-induced improving effect on the gel formability of myosin. The cross-linking of myosin and actomyosin molecules produces a mechanically stable gel, and this structure may play a crucial role in the binding of a cured meat product.

The objective of the present study was to find roles, on a molecular scale, of regulatory proteins represented by native tropomyosin and of structurally different regions of myosin molecule, in the formation of a mechanically self-supporting gel or network. To achieve those objectives we have studied the heatinduced gelation of reconstituted Factomyosin systems in the presence or absence of the native tropomyosin and F-actin-myosin derived subfragments.

\section{MATERIALS AND METHODS}

Preparation of myosin and myosin derived subfragments. Myosin was prepared from the muscles of the hind legs and back of rabbits essentially as described by Perry. ${ }^{20)}$ The stock myosin solution $(20 \mathrm{mg} / \mathrm{ml})$ was stored in $0.6 \mathrm{M}$ $\mathrm{KCl}$ at $0^{\circ} \mathrm{C}$.

Heavy meromyosin (HMM) and light meromyosin (LMM) were prepared by digesting a myosin solution $(10 \mathrm{mg} / \mathrm{ml})$ in $0.6 \mathrm{M} \mathrm{KCl}$ and $20 \mathrm{mM} \mathrm{K}$-phosphate buffer (pH 7.0) for $10 \mathrm{~min}$ at $25^{\circ} \mathrm{C}$ in the presence of $1 \mathrm{mM} \mathrm{MgCl}_{2}$ and $1 \mathrm{~mm}$ dithiothreitol (DTT, Sigma) with $\alpha$-chymotryp$\sin$ (Sigma) at a final concentration of $0.03 \mathrm{mg} / \mathrm{ml} .^{2,21,22)}$ Digestion was inhibited with phenylmethyl sulfonyl fluoride (PMSF, Sigma) at concentration of $0.5 \mathrm{~mm}$. HMM was isolated in the supernatant by dialysis against $50 \mathrm{~mm}$ $\mathrm{KCl}$ and $10 \mathrm{~mm} \mathrm{K-phosphate} \mathrm{buffer} \mathrm{(pH} \mathrm{7.0)} \mathrm{and} \mathrm{further}$ purified by fractionation of $57 \%$ saturated ammonium sulfate and by subsequent column chromatography on DEAE-cellulose. ${ }^{21)}$ From the insoluble residue of the HMM preparation, LMM was made by the method reported by Samejima et ll. $^{23)}$

Chymotryptic subfragment 1 (S-1) and myosin rod (Rod) were prepared according to Weeds and Taylor ${ }^{21}$ and Weeds and Pope $^{22)}$ from insoluble filaments in $0.12 \mathrm{M}$ $\mathrm{KCl}$ and $20 \mathrm{mM} \mathrm{K}$-phosphate buffer $(\mathrm{pH} 7.0)$ in the presence of $1 \mathrm{mM}$ ethylene diaminetetraacetic acid (EDTA) and $1 \mathrm{~mm}$ DTT. A myosin suspension $(10 \mathrm{mg} / \mathrm{ml})$ was digested at $25^{\circ} \mathrm{C}$ for $10 \mathrm{~min}$ with $0.1 \mathrm{mg} / \mathrm{ml} \alpha$-chymotrypsin and the reaction was stopped with PMSF to a final concentration of $0.5 \mathrm{~mm}$. Insoluble material was removed by centrifugation, and the supernatant was purified by DEAE-column chromatography. From the insoluble resi- due, Rod was prepared using the ethanol fractionation procedure. $^{23)}$ Electrophoretic patterns of our protein samples were illustrated in our preceding papers. ${ }^{9,10)}$

Preparation of actin. Rabbit skeletal actin was extracted from acetone powder in the cold with a solution containing $0.2 \mathrm{~mm}$ ATP and $5 \mathrm{~mm}$ Tris- $\mathrm{HCl}(\mathrm{pH} \mathrm{7.8)}$. The crude extract of $\mathrm{G}$-actin was polymerized in $50 \mathrm{mM} \mathrm{KCl}$ and $5 \mathrm{~mm}$ Tris- $\mathrm{HCl}(\mathrm{pH} 8.0)$ at $25^{\circ} \mathrm{C}$. For further purification, the cycle of polymerization, centrifugation and dialysis was repeated, except for the use of $0.6 \mathrm{M} \mathrm{KCl}$ instead of $50 \mathrm{~mm} \mathrm{KCl}$ for the polymerization. Actin was stored in Gform. When needed, it was converted to F-form in $50 \mathrm{~mm}$ $\mathrm{KCl}$ and $5 \mathrm{~mm}$ Tris- $\mathrm{HCl}(\mathrm{pH} 8.0)$ at $25^{\circ} \mathrm{C}$ and sedimented at $30,000 \mathrm{rpm}$. Almost no tropomyosin contamination was observed in the sodium dodecylsulfate (SDS)-polyacrylamide gel electrophoretograms of our actin preparation.

Reconstituted actomyosin, acto-HMM and acto-S-1 were prepared by mixing appropriate amounts of F-actin to myosin and subfragments by weight. These mixtures were taken to $5 \mathrm{mg}$ total protein $/ \mathrm{ml}, 0.6 \mathrm{M} \mathrm{KCl}$ and kept overnight in ice before use and $20 \mathrm{~mm} \mathrm{~K}$-phosphate buffer ( $\mathrm{pH}$ 6.0, final conc.) was added to adjust the $\mathrm{pH}$ value prior to the heat treatment.

Determination of binding of myosin to F-actin and stoichiometry of the binding. Binding of myosin and its subfragments S-1 and HMM to F-actin was monitored by the light scattering intensity at an angle of $90^{\circ}$ of the reaction mixture in $0.6 \mathrm{M} \mathrm{KCl}$ and $20 \mathrm{~mm}$ phosphate $(\mathrm{pH}$ $6.0)$ at $25^{\circ} \mathrm{C}$ and the change in the intensity at $535 \mathrm{~nm}$ was recorded by using a fluorescence spectrophotometer (Hitachi 650-60).

Quantitative measurements of myosin binding to $\mathrm{F}$ actin were performed using the preparative ultracentrifuge to separate bound myosin from free. Four $\mathrm{ml}$ of the mixture of myosin and actin described in the previous section was immediately centrifuged at $100,000 \mathrm{~g}$ for $120 \mathrm{~min}$ before use. The supernatant (about $1 \mathrm{ml}$ ) was carefully removed and the protein concentration was measured by an appropriate method which will be described later in this section. The protein concentration thus measured represents the sum of all proteins remaining in the supernatant after removal of the polymeric species by sedimentation. This value will, therefore, include not only the unbound myosin but also any unpolymerized actin. Hence, it was essential to determine the amount of such contaminants in the supernatant and to correct the protein concentration for their presence. The correction of unpolymerized actin was determined in each experiment by running the amount of actin used in the reaction mixture in a separate cell along with the acto-enzyme samples.

The actin and myosin concentration in each sample were also estimated by densitometry of the actin band and the heavy chain of myosin band by means of a Toyo Kagaku Densitometer DMU-33C. Optical density 
measurements were found to be linear between 0 and $15 \mu \mathrm{g}$ for actin, and 0 and $15 \mu \mathrm{g}$ for myosin heavy chain, respectively.

The stoichiometry of the binding of myosin to actin under our experimental conditions was obtained by measuring the protein concentration of the myosin and actin in the supernatant of the centrifuged reaction mixture as a function of myosin concentration at constant actin concentration.

Preparation of native tropomyosin. Native tropomyosin was isolated by the method of Ebashi et al. ${ }^{24,25)}$ from minced rabbit skeletal muscle, free of myosin residues. The purity of native tropomyosin preparations were routinely checked with measurements of $\mathrm{Mg}^{2+}$-EGTA ATPase activity, SDS-polyacrylamide gel electrophoresis and by superprecipitation assay. ${ }^{25}$

Sodium dodecyl sulfate gel electrophoresis. Electrophoresis on polyacrylamide gels $(7.5 \%)$ was carried out as described by Weber and Osborn. ${ }^{26)}$ Samples in $0.01 \mathrm{M}$ Na-phosphate ( $\mathrm{pH} 7.0$ ) were boiled for $2 \mathrm{~min}$ in $1 \%$ SDS and $0.1 \% 2$-mercaptoethanol before being applied to the gel. Electrophoresis was carried out for $4 \mathrm{hr}$ at $8 \mathrm{~mA} /$ tube $(70 \mathrm{~mm})$. The gels were then simultaneously fixed and stained by diffusion in $45.4 \%$ methanol and $9.2 \%$ acetic acid, containing $0.25 \%$ Coomassie brilliant blue stain; destaining was done by diffusion in $5 \%$ methanol and $7.5 \%$ acetic acid.

Scanning electron microscopy (SEM). Scanning electron microscopic observations were made on the heat-set gels of myosin and reconstituted actomyosin, using a JEOL JSM-T 200 scanning electron microscope.

Gelation. The gelation of myosin and its proteolytic subfragments in the presence or absence of varying amount of F-actin or F-actomyosin, or that of actomyosin in the presence or absence of native tropomyosin was measured by the use of the band type viscometer as reported previously. ${ }^{5}$ ) The gelation was induced by heating the sample solutions in $0.6 \mathrm{M} \mathrm{KCl}$ at $\mathrm{pH} 6.0$ and $65^{\circ} \mathrm{C}$ for $20 \mathrm{~min}$ and measured as changes in rigidity.

Superprecipitation. Superprecipitation experiments were carried out in a $10 \mathrm{~mm}$ glass cell and changes in turbidity were measured at $660 \mathrm{~nm}^{11)}$ with a Hitachi 101 spectrophotometer.

Protein concentration. Protein concentration was determined by the biuret or UV absorption method as described in a previous paper. ${ }^{7)}$ For conversion of the protein concentration from the by weight basis to a molar basis, molecular weights of the proteins used were taken as 480,000 for myosin, 27) 340,000 for $\mathrm{HMM},{ }^{27)}$ 120,000 for $S-1,{ }^{22)} 220,000$ for $\operatorname{Rod}^{22)} 140,000$ for $\mathrm{LMM}^{22)}$ and 42,000 for actin. ${ }^{28)}$

\section{RESULTS AND DISCUSSION}

\section{Some reinvestigations on actin and on myosin-} actin interaction

In their studies on the heat-induced gelation of myosin in the presence of actin, Yasui et $a l .{ }^{7)}$ stated that actin was added to the reaction mixture in the G-form to induce simultaneously polymerization of G-actin to F-form and binding of F-actin with myosin in the system. The state of actin upon addition may have a large effect on the results because actin polymerizes very slowly in high salt and low temperature. ${ }^{29)}$ Also, it is not clear whether the amount of unpolymerized protein species in the myosin-actin system can be corrected on the basis of the amount of actin not sedimented at $100,000 \mathrm{~g}$ for $120 \mathrm{~min}$ in tubes containing actin alone. Myosin is known to accelerate polymerization of actin into the F-form, and the presence of myosin may greatly change the ratio of F- to G-actin. These concerns were the first to be examined in this work, so that the subsequent results would be correct in the quantitative sense.

Figure 1 indicates the effect of the state of actin upon addition on the $\mathrm{G} \rightleftharpoons \mathrm{F}$ transformation equilibria under our experimental conditions. In the absence of myosin [Fig. 1(A)], the amount of polymerized actin was found to be about $50 \%$ of the total amount of added Gactin at a concentration of $0.3 \sim 0.5 \mathrm{mg} / \mathrm{ml}$, while it was about $80 \%$ at a concentration of $5 \mathrm{mg} / \mathrm{ml}$. As reported by Yasui et al., ${ }^{7)}$ almost the same results were obtained when F-actin was used in place of G-actin at the above mentioned concentration range. However, at actin concentrations in between both G- and F-actin showed significant concentration dependence in the $\mathrm{G} \rightleftharpoons \mathrm{F}$ equilibria, and between $0.5 \mathrm{mg} / \mathrm{ml}$ and $5.0 \mathrm{mg} / \mathrm{ml}$, the amount of polymerized actin was always lower in the case of G-actin than was in the case of F-actin. This may imply that the $\mathrm{G} \rightleftharpoons \mathrm{F}$ equiribrium over the concentration range specified above is reached at different stages with F-and G-form, respectively.

Figure 1(B) demonstrates the result of a 


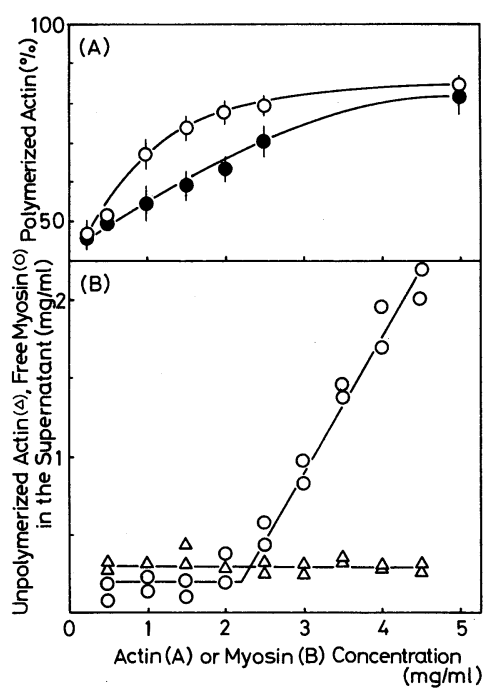

FIG. 1. Concentration Dependence of $\mathrm{G} \rightleftharpoons \mathrm{F}$ Transformation of Actin (A) and Stoichiometry of Myosin-Actin Interaction (B).

(A): Varying concentration of F- (O) or G- (O) actin solutions in $0.6 \mathrm{M} \mathrm{KCl}$ incubated overnight in ice. Amount of polymerized actin was calculated from protein concentration in the supernatant after centrifugation at $100,000 \mathrm{~g}$ for $120 \mathrm{~min}$. Bars in the figure are standard deviations from three or four experiments.

(B): Incubation medium containing varying concentrations of myosin and $1 \mathrm{mg} / \mathrm{ml} \mathrm{F}$-actin in $0.6 \mathrm{M} \mathrm{KCl}$ was incubated overnight in ice. Concentrations of myosin $(\mathrm{O})$ and actin $(\triangle)$ were determined by densitometry on SDSpolyacrylamide gel electrophoretograms of the supernatants after centrifugation at $100,000 \mathrm{~g}$ for $120 \mathrm{~min}$.

typical experiment on the determination of stoichiometry of myosin binding to F-actin, as well as on the influence of the presence of myosin on the $\mathrm{G} \rightleftharpoons \mathrm{F}$ transformation. In this experiment, we used $1 \mathrm{mg} / \mathrm{ml}$ of actin throughout and added varying concentrations of myosin. After overnight incubation in ice, each sample mixture was centrifuged, and the supernatant was subjected to electrophoresis.

Irrespective of the change in myosin concentration of the system, the amount of unpolymerized actin (about $0.25 \sim 0.3 \mathrm{mg} / \mathrm{ml}$ ) remained constant. A very small amount of myosin was detectable in the supernatant and stayed at the constant level unitl its concentration in the system became $2.1 \mathrm{mg} / \mathrm{ml}$. Beyond this breaking point, an abrupt increase in myosin concentration took place, indicating saturation of myosin binding to F-actin. At the same time, it seem to be clear that $\mathrm{G} \rightleftharpoons \mathrm{F}$ transformation is kept at the same level in the presence or absence of myosin under the present experimental conditions.

After the correction for unpolymerized actin, the stoichiometry of myosin binding to Factin has been calculated to be 2.96 by weight which, in terms of molar basis, corresponds to about 0.26 . The same molar ratio has recently been reported on natural actomyosin ${ }^{30)}$ extracted from chicken gizzard and on S-1 or myosin binding with $\mathrm{F}$-actin at an ionic strength of $0.1{ }^{31)}$ Since the established stoichiometry of myosin-actin interaction at physiological conditions is believed to be at a molar ratio of $0.5,{ }^{27,31)}$ it is important to recognize that there is no contradiction between the result presented here and those of previous work ${ }^{27,31)}$ on acto-myosin and acto-HMM.

Presumably because earlier electron micrographs of F-actin decorated with myosin or HMM are single filaments with an arrow head structure, ${ }^{33,34)}$ actomyosin in solution is thought to exist in this form only. Some evidence that the two heads of myosin indeed cross-link actin filaments had been provided by Abe and Maruyama ${ }^{35,36)}$ who demonstrated on the basis of their dynamic viscoelasticity study of acto-HMM in solution that actin and HMM can together form a gel, and by Trinick and Offer ${ }^{37)}$ who showed the nature of the cross-links by electronmicroscopy. The interparticular interaction is reported to be highest when the molar ratio of HMM to actin is less than 0.5 . In addition, when we consider that the interaction is endothermic and considerably inhibited by high ionic strength, the actomyosin solution used in Fig. 1(B) may possibly have a network structure with a local aggregation $^{35,37)}$ which will limit the number of both intra- and interfilamental interaction sites. It is conceivable that such a situation could affect the stoichiometry of the binding.

Augmentation of the heat-induced gel formability of myosin by the presence of F-actin 


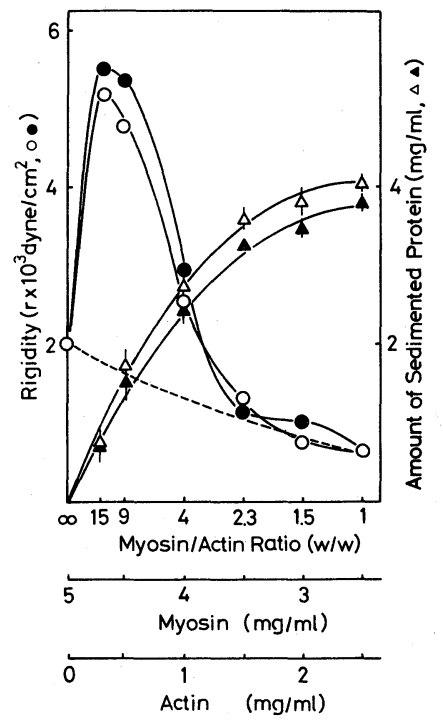

FIG. 2. Changes in the Rigidity of the Heat-induced Actomyosin Gels and in the Amount of Sedimentable Proteins as a Function of Myosin/Actin Weight Ratios.

The samples $(5 \mathrm{mg} / \mathrm{ml})$ dissolved in $0.6 \mathrm{M} \mathrm{KCl}$ and $20 \mathrm{~mm}$ phosphate buffer ( $\mathrm{pH}$ 6) were incubated at $65^{\circ} \mathrm{C}$ for 20 min. Amount of sedimented proteins before heating was determined from the protein concentration in the supernatant after centrifugation at $100,000 \mathrm{~g}$ for $120 \mathrm{~min}$. F- $(O$, rigidity; $\triangle$, sedimentable protein) or G- $(\bullet$, rigidity; $\boldsymbol{\Delta}$, sedimentable protein) actin was added to myosin (dotted line) solution (for details refer to MATERIALS AND Methods). Bars in the figure are standard deviations from the three or four experiments.

Figure 2 illustrates the result of experiments of the heat-induced gelation of myosin in the presence of F-actin. The heat-induced gel formability of myosin as measured by rigidity is biphasically influenced as the amount of Factin in the system increases. The rigidity increased from $2,000 \mathrm{dyn} / \mathrm{cm}^{2}$ (myosin alone) to more than $5,000 \mathrm{dyn} / \mathrm{cm}^{2}$ until a myosin to actin ratio of 15 by weight was reached, and with a ratio of less than 9 the amount of rigidity decreased greatly. This quantitative relationship is substantially the same as the one observed with G-actin (Fig. 2). Simultaneous determination of sedimentable protein, which reflects roughly actomyosin complex formation in the system shows that about $15 \%$ of the total protein was sedimentable as the complex at the maximum amount of rigidity. Also, the curve for the complex formation seem to be fairly consistent with those for $\mathrm{G} \rightleftharpoons \mathrm{F}$ transformtion of actins in Fig. 1(A).

The treatments described in our preceding paper, ${ }^{8)}$ which block the interaction between myosin and actin, were also applied and found to supress to the great extent or completey abolish the augmentation of gel-fomability observed in the presence of F-actin. The results are not shown here, because they have coincided with those in ref. 8 . The results thus far obtained have not only offered satisfactory answers for the concerns introduced at the beginning of this section, but also verified the conclusion of our previous studies, ${ }^{7,8)}$ i.e., the actin-induced improving effect on thermogelling properties of myosin is dependent exclusively of the relatively small amount of Factomyosin formed in the myosin-actin system.

\section{The heat-induced gelation of myosin in the presence of F-actomyosin}

Above mentioned interpretations, together with our earlier findings ${ }^{5 \sim 10}$ ) enable us to postulate that, in enhancement of the heatinduced gelation, an appropriate amount of actomyosin complex formed by myosin binding with $\mathrm{F}$-actin is capable of providing extra cross-linkages for the framework through linking of the tail regions between free myosin and bound myosin with F-actin. This implies that, in the mixture of myosin and F-actin, only a whole molecule of myosin can take part in the effect, for the head region of myosin has an ability to bind with F-actin, but not to create a framework, ${ }^{9,10)}$ and tail region has the potential to produce gels, but not to bind with Factin.

To substantiate the reasoning, experiments on the effect of actomyosin in the heat-induced gelation of myosin were performed using a system composed of myosin and actomyosin. The actomyosin used in this experiment was obtained from the pellet prepared from a myosin-F-actin mixture having the weight ratio of $10: 1$. Myosin/actin ratio $(w / w)$ of this 


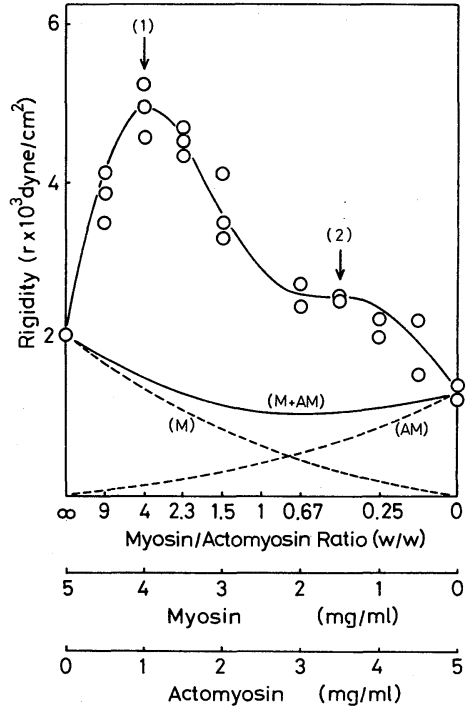

FIG. 3. Changes in the Rigidity of the Heat-induced Gels of Myosin-F-Actomyosin Mixtures as a Function of Myosin/F-Actomyosin Weight Ratios.

Conditions were the same as in Fig. 2. (M), myosin alone; (AM), F-actomyosin alone; $(\mathrm{M}+\mathrm{AM})$, sum of $(\mathrm{M})$ plus (AM).

actomyosin was found to be 2.96 according to densitometric analysis of the SDS-polyacrylamide gel electrophoretogram, showing that the actomyosin sample has been saturated with myosin (Fig. 1(B)). The result is shown in Fig. 3.

If buffer alone was added in place of actomyosin or myosin, the rigidity measurements were those expected for dilution of the myosin [dotted line (M) in Fig. 3] or of the actomyosin [dotted line (AM) in Fig. 3]. If there were no interaction between the two molecular species, the rigidity of the system upon heating would be around the sum of rigidity values of $\mathrm{F}$-actomyosin and myosin at concentrations specified in the abscissa. Actually, however, keeping the total protein concentration at $5 \mathrm{mg} / \mathrm{ml}$, rigidity values measured as a function of the weight ratio of myosin F-actomyosin increased from about 2,000 $\mathrm{dyn} / \mathrm{cm}^{2}$ (myosin alone) to the value of as much as about $5,000 \mathrm{dyn} / \mathrm{cm}^{2}$ until the ratio reached 4 . With a ratio less than 4 the rigidity value decreased greatly to about 2,200 dyn/ $\mathrm{cm}^{2}$ down to a ratio of 0.7 . The amount of rigidity remained constant until the ratio was in the vicinity of 0.25 and then dropped to about 1,200 dyn $/ \mathrm{cm}^{2}$ (actomyosin alone). This rigidity profile looks very similar to that shown in Fig. 2 and ref. 7, and presents clearcut evidence for the primary role of actomyosin in the actin-induced improving effect on the heat-induced gelation of myosin. The gel formability of the myosin-actomyosin system is maximally enhanced when it consists of $80 \%$ of free myosin and $20 \%$ of fully decorated $\mathrm{F}$-actomyosin under these experimental conditions.

Figure 4 shows SEM pictures of gels taken from the heat-set gels specified by arrows (1) and (2) in Fig. 3. (A) corresponds to the gel of myosin (not specified in Fig. 3), (B) to the gel of the myosin-actomyosin mixture at the ratio of 4 [arrow (1) in Fig. 3], (C) to the one at the ratio of 0.43 [arrow (2) in Fig. 3], and (D) to the gel of actomyosin (not specified in Fig. 3).

The micrographs indicate a series of changes in morphology of the gels arising as a consequence of increased actomyosin concentration. Such heat-induced networks vary in pore size distirubiton, and in network strand shape and thickness, depending apparently on the model of protein aggregation, ranging from assemblies of irregular protein clumps (A) to filamentous strands with entangled clusters (D). Between the two, SEM pictures for (B) and (C) give us the impression of network based on F-actomyosin thin filaments of various degrees of persistence, and cross-linked to a different extent. Irregular chains of myosin, which are characterized by inhomogenous granular particles linked by smooth shafts, turned out to be cross-linked along the thiner and smoother network strands in the myosinactomyosin mixture at the weight ratio of 4(B). With a decrease in the myosin/actomyosin ratio to 0.43 , the system gave a micrograph $(\mathrm{C})$ in which the network structure of (B) and Factomyosin itself (D) coexist. The same filaments of protein seem to underly the structures in (B), (C) and (D), but in (D) there is a higher occurrence of side to side cross-linking be- 

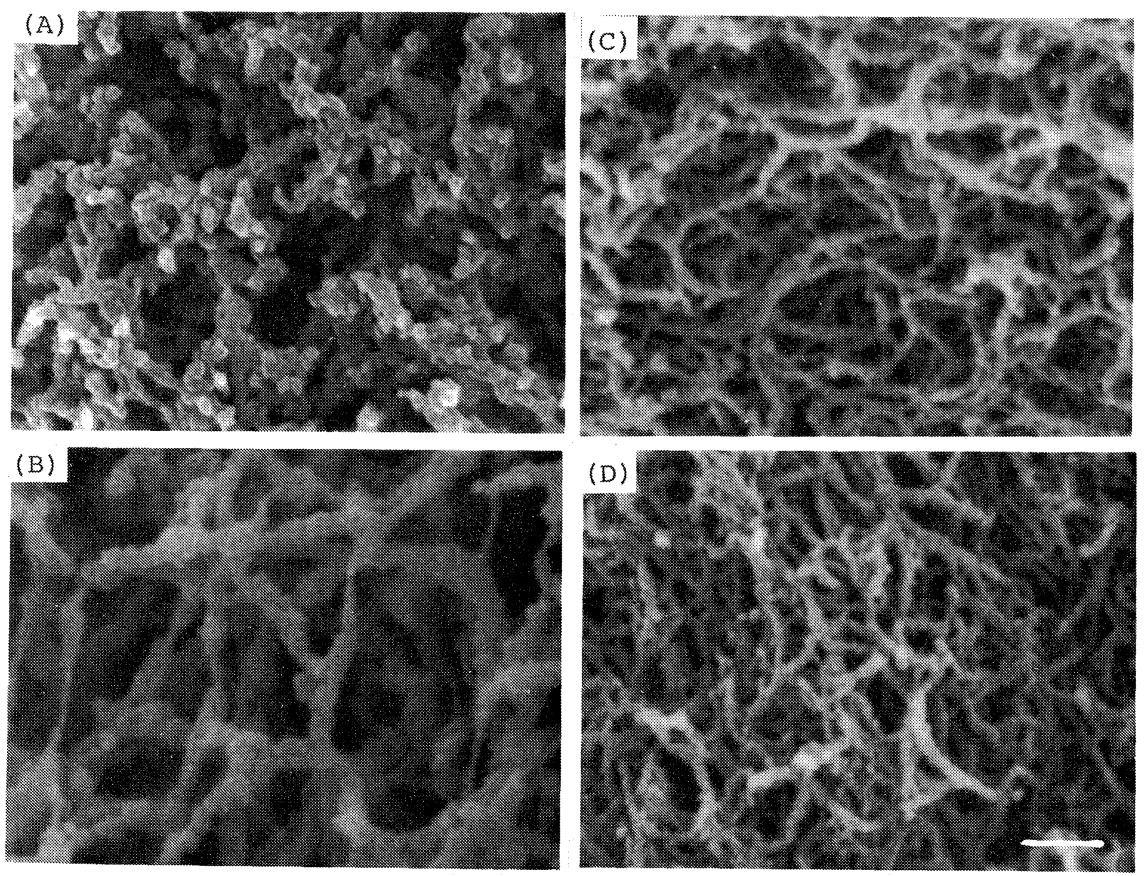

FIG. 4. Scanning Electron Micrographs for Heat-set Gels of Myosin (A), Myosin/F-Actomyosin Mixtures $[(B)$ and $(C)]$ and F-Actomyosin (D) Formed at $65^{\circ} \mathrm{C}$ in $0.6 \mathrm{M} \mathrm{KCl}$.

The weight ratios of myosin to F-actomyosin were $4(\mathrm{~B})$ and 0.43 (C), respectively. The effect of F-actomyosin at those ratios on the heat-induced gelation of myosin are indicated by arrows (1) and (2) in Fig. 3. Bar length is $1 \mu \mathrm{m}$.

tween filaments. In (B), on the other hand, cross-linking between free myosin and bound myosin seems to prevail.

It is worth noting that there are apparent indications in the SEM views from (B) to (D) that the filamentous form of F-actomyosin does survive even in the heat-set gels in the presence of myosin. F-Actin alone does not form a gel upon heating, but instead coagulates, and any network present initially ${ }^{37)}$ collapses into a compact "string of beads" like denatured actin globules.")

The heat-induced gelation of myosin derived subfragments ( $S-1, H M M$, Rod and LMM) in the presence of F-actin or F-actomyosin

What the electron microscope can not do is to indicate precisely the structural form each molecule adopts when it becomes part of the gel network. To confirm the validity of the interpretation of rigidity profiles and SEM pictures, thermogelling properties of myosin derived subfragments (S-1, HMM, Rod and LMM) were investigated in the presence of Factin [Fig. 5(A)] and F-actomyosin [Fig. 5(B)]. Rigidity profiles of each system have been compared with those for myosin under the same conditions [Figs. 2 and 3]. No enhancement of the heat-induced gelation was observed when we added F-actin to the systems containing these subfragments of myosin in $0.6 \mathrm{M} \mathrm{KCl}$ and at $\mathrm{pH} 6.0$ [Fig. 5(A)]. Light scattering experiments [Fig. $5(\mathrm{C})$ ] conducted prior to the heat treatments clearly demonstrated that both the S-1 and HMM (single and double headed subfragments) were capable of binding with F-actin. On the other hand, the Rod and LMM, a whole and part of the helical tail region of myosin molecule, showed no sign of interaction with $\mathrm{F}$-actin [Fig. 5(B)], obviously due to the lack of a binding site(s) which resides only in the heavy 


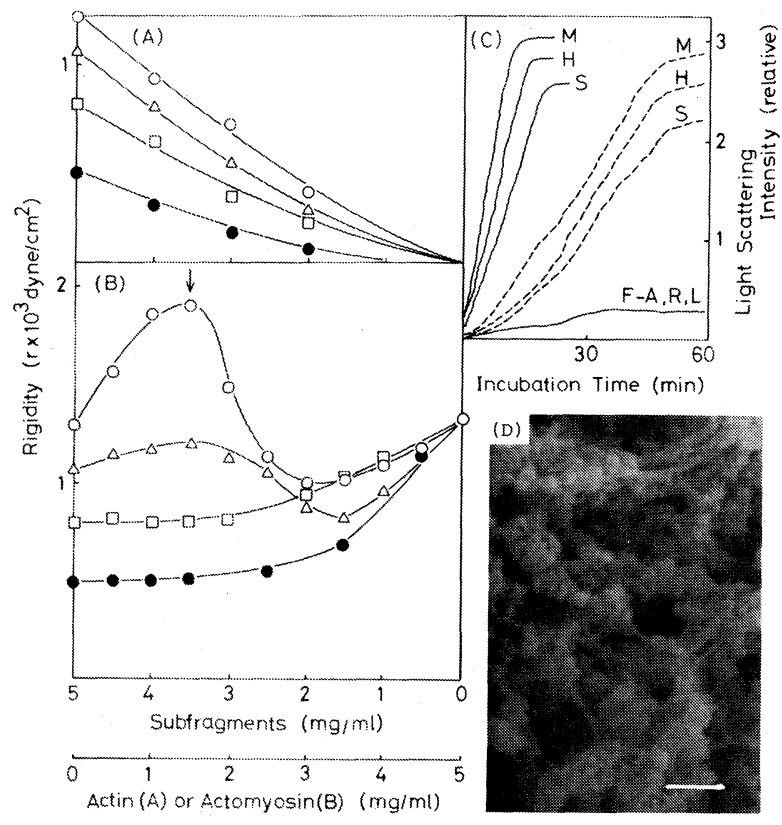

FIG. 5. Effects of F-Actin and F-actomyosin on the Heat-induced Gelling Properties of Myosin-derived Subfragments.

Changes in the rigidity of the heat-induced gels of F-actin-myosin subfragments systems (A) and of Factomyosin-myosin subfragments systems $(B)$ as a function of the weight ratios of myosin subfragments to Factin in (A) and of myosin subfragments to F-actomyosin in (B), respectively. (C) and (D), respectively, illustrates interactions of myosin and its subfragments with actin and a SEM picture of the heat-set gel of rodF-actomyosin system shown by an arrow in (B). Bar length is $1 \mu \mathrm{m}$. (A) and (B), conditions were the same as Fig. 2. (O), rod; $(\triangle)$, LMM; $(\square)$, HMM and (O), S-1. (C), solid lines: F-actin (F-A) and myosin (M), HMM (H), S-1 (S), rod (R) and LMM (L) were mixed by mol [(F-A, 7.2 $\mu \mathrm{M}) /(\mathrm{M})$ or subfragments $=3$, but in the case of $\mathrm{S}-1,1.5]$ in $0.6 \mathrm{M} \mathrm{KCl}$ at $\mathrm{pH} 6.5 \sim 7.0$ and light scattering intensity at $535 \mathrm{~nm}$ was measured with time of incubation at $25^{\circ} \mathrm{C}$. Dotted lines: G-actin was used in place of F-actin.

chain of the head region. ${ }^{27,32)}$

Although the S-1 and HMM can form the complex with F-actin [Fig. 5(C)], they are devoid of the necessary segment of the tail for the production of framework, so that they can not connect in a network. In the case of the Rod and LMM, they themselves can cause the network system to gel, but because of the absence of head region, they do not have ability to complex with F-actin [Fig. 5(C)]. Thus, in contrast to myosin (Fig. 1), neither headed nor helical subfragments exhibited any remarkable augmentation of the heat-induced gelation in the presence of actin [Fig. 5(A)]. All results shown in Fig. 5(A) are simple dilution effects on the gelation of respective subfragments upon heating. ${ }^{9,10)}$
When mixed with F-actomyosin in place of F-actin as has been done in Fig. 2, the actomyosin was found to reveal a significant enhancing effect on the heat-induced gelation of the Rod and on that of the LMM as well, though to lesser extent. Contrarily, almost no influence over the heat-induced gelation of S-1 and HMM was observed by the introduction of F-actomyosin to the system [Fig. 5(B)].

A reasonable explanation for the results is given by the idea that cross-linking between free and bound myosin molecules in the system is effectively initiated only through interactions between tail (LMM) portions of the two species of myosin molecules. Thus, even the Rod and LMM, both of which possess potential ability to form network, ${ }^{9,10)}$ are able to 
join the gelation of actomyosin upon heating, whereas the S-1 and HMM fail to participate in the gelation due to the lack of LMM segment. These findings unerringly implicate that the actomyosin-induced improving effect on the heat-induced gel formability of myosin is achieved by cross-linking myosin aggregated through tail regions of free and bound myosin molecules to produce a mechanically more stable gel than does myosin alone, and the primary importance of the role of LMM segment of myosin molecules in the development of the thermogelling properties ${ }^{9,10)}$ of this protein. A SEM picture of the heat-set gel taken from the Rod-actomyosin system [Fig. 5(D)], which shows gathering of Rod clusters along F-actomyosin filaments, may present additional evidence to reinforce the interpretation stated above.

\section{Effect of native tropomyosin}

In vertebrate muscles, troponin and tropomyosin are apparently the only regulatory proteins and the control is therefore actinlinked. ${ }^{38)}$ Naturally occurring thin filaments (actin filaments) in myofibrils contain one mol of tropomyosin and troponin complex per 7 mol of actin subunits. ${ }^{24,27,38)}$ Thus it is plausible to presume that these regulatory proteins might participate in the heat-induced gelation of natural actomyosin or more practically in cured muscle mince. In our very recent study ${ }^{11)}$ we presented an extensive survey on the gelation comparing natural actomyosin (NAM) with desensitized actomyosin (DAM, natural actomyosin from which regulatory proteins were removed by repeated washing with a low ionic strength solution ${ }^{39}$ ). This investigation showed that the heat-induced gelation of NAM was not influenced by the presence or absence of regulatory proteins.

In the present study, these observations have been extended to reconstituted actomyosin which consisted of purified myosin and actin freed from regulatory proteins, and the results are summarized in Fig. 6. As shown in Fig. 6 (inset), when added to the reconstituted actomyosin without Ca-sensitivity ${ }^{24,25,38,39)}$ due

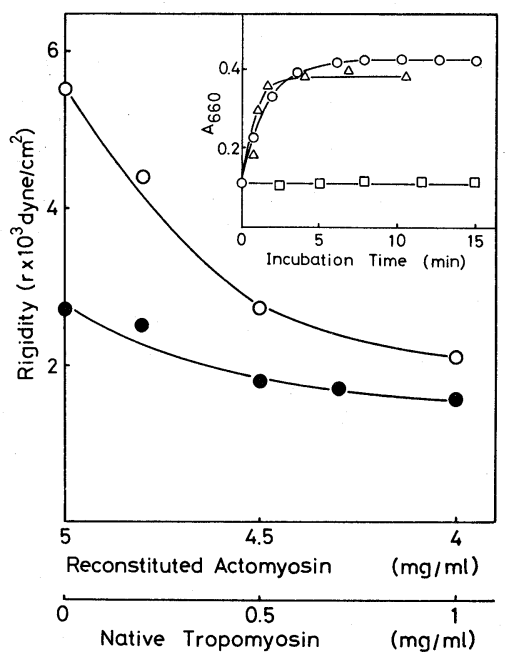

FIG. 6. Effect of Native Tropomyosin on the Heatinduced Gelation of Actomyosins.

Actomyosins in which the weight ratios of myosin to actin were $9(\bigcirc)$ and $4(\bigcirc)$ were used in this experiment. Inset: Effect of native tropomyosin on $\mathrm{Ca}^{2+}$-sensitivity of reconstituted actomyosin judged from superprecipitation. Reaction mixture contained $0.4 \mathrm{mg} / \mathrm{ml}$ reconstituted actomyosin $(\mathrm{M} / \mathrm{F}-\mathrm{A}=4$, w/w) $0.1 \mathrm{mg} / \mathrm{ml}$ native tropomyosin, $0.06 \mathrm{M} \mathrm{KCl}, 40 \mathrm{~mm}$ Tris-maleate (pH 7.0), $1 \mathrm{~mm}$ $\mathrm{MgCl}_{2}$ and $1 \mathrm{~mm} \mathrm{ATP}$ in the presence $(\bigcirc)$ or absence $(\square)$ of $1 \mathrm{~mm}$ EGTA. $(\triangle)$, reconstituted actomyosin alone in the same conditions in the presence of $1 \mathrm{mM}$ EGTA. Incubation was carried out at $25^{\circ} \mathrm{C}$.

to the absence of regulatory proteins, native tropomyosin which is composed of tropomyosin and troponins, troponin- $\mathrm{T},-\mathrm{I}$ and $-\mathrm{C}$, at a molar ratio of $1: 1: 1: 1,{ }^{27)}$ complexed with F-actomyosin and the system turned out to be Ca-sensitive. Despite its definite incorporation into the F-actomyosin system (Fig. 6, insert), varying amounts of the native tropomyosin added to the two reconstituted actomyosin systems (weight ratios of myosin to actin were 9 and 4, respectively) was found to exert no influence on the heat-induced gel formability of these systems.

There is insufficient evidence to propose that regulatory proteins are implicated in the heatinduced gelation mechanism of F-actomyosin, although the results by Nakayama and Sato ${ }^{14,15)}$ and Cheng et al. $^{40)}$ suggest that incorporation of regulatory proteins into Factomyosin filaments will allow the improve- 
ment of rheological properties of the heat-set gels of the system. From earlier studies ${ }^{11)}$ and from the experiments shown in Fig. 6, it is reasonable to discount the involvement of native tropomyosin, which represents regulatory proteins in muscle, in the heat-induced gelation of the myosin-actomyosin system. Thus the previous studies by Nakayama and Sato $^{14,15)}$ and Cheng et al. ${ }^{40)}$ must be reevaluated on the basis of the findings presented in this and our preceding ${ }^{11)}$ paper, as well as of the up-to-date knowledge on muscle biochemistry.

The results obtained in this study lead us to the following conclusion: Actin-induced improving effects on the heat-induced gel formability of myosin as expressed by rigidity results solely from $\mathrm{F}$-actomyosin formation in the myosin-actin system. The effect, therefore, depends more directly on the myosin (free)/ actomyosin (fully decorated) ratio by weight in the system, rather than on myosin/actin ratio. When the ratio of 4 is reached, the effect is optimally developed in the system under the standard conditions in this study (in $0.6 \mathrm{M} \mathrm{KCl}$ at $\mathrm{pH} 6.0$ and $65^{\circ} \mathrm{C}$ for $20 \mathrm{~min}$ ).

This also suggests that binding as well as water-holding properties of cured meat product are determined only by the ratio of free myosin to F-actomyosin with or without native tropomyosin in the solubilized myofibrillar proteins during the curing process prior to heat-treatment of comminuted meat products.

Acknowledgments. The authors express their sincere appreciation to Professor Dr. F. H. Wolfe of University of Alberta, Canada for reading this manuscript.

This works was supported in part by a Grant-in-Aid for Scientific Research (547113), 1981, from the Ministry of Education, Science and Culture of Japan.

\section{REFERENCES}

1) T. Fukazawa, Y. Hashimoto and T. Yasui, J. Food Sci., 26, 331 (1961).

2) T. Fukazawa, Y. Hashimoto and T. Yasui, J. Food Sci., 26, 541 (1961).

3) T. Fukazawa, Y. Hashimoto and T. Yasui, J. Food Sci., 26, 550 (1961).

4) K. Samejima, Y. Hashimoto, T. Yasui and T.
Fukazawa, J. Food Sci., 34, 242 (1969).

5) T. Yasui, M. Ishioroshi, H. Nakano and $K$. Samejima, J. Food Sci., 44, 1201 (1979).

6) M. Ishioroshi, K. Samejima and T. Yasui, J. Food Sci., 44, 1280 (1979).

7) T. Yasui, M. Ishioroshi and K. Samejima, J. Food Biochem., 4, 61 (1980).

8) M. Ishioroshi, K. Samejima, Y. Arie and T. Yasui, Agric. Biol. Chem., 44, 2185 (1980).

9) K. Samejima, M. Ishioroshi and T. Yasui, J. Food Sci., 46, 1412 (1981).

10) M. Ishioroshi, K. Samejima and T. Yasui, J. Food Sci., 47, 114 (1982).

11) K. Samejima, M. Ishioroshi and T. Yasui, Agric. Biol. Chem., 46, 535 (1982).

12) G. R. Hegarty, L. J. Bratzler and A. M. Pearson, $J$. Food Sci., 28, 663 (1963).

13) T. Nakayama and Y. Sato, Agric. Biol. Chem., 35, 208 (1971).

14) T. Nakayama and Y. Sato, J. Texture Studies, 2, 75 (1971).

15) T. Nakayama and Y. Sato, J. Texture Studies, 2, 475 (1971).

16) R. Tsai, R. G. Cassens and E. J. Briskey, J. Food Sci., 37, 286 (1972).

17) J. J. Macfarlane, G. R. Schmidt and R. H. Turner, $J$. Food Sci., 42, 1603 (1977).

18) D. E. Siegel and G. R. Schmidt, J. Food Sci., 44, 1686 (1979).

19) D. G. Siegel and G. R. Schmidt, J. Food Sci., 44, 1129 (1979)

20) S. V. Perry, "Methods in Enzymology," Vol. II, ed. by S. P. Colowick and N. O. Kaplan, Academic Press, Inc., New York, 1955, p. 582.

21) A. G. Weeds and R. S. Taylor, Nature (London), 257, 54 (1975).

22) A. G. Weeds and B. Pope, J. Mol. Biol., 111, 129 (1977).

23) K. Samejima, K. Takashashi and T. Yasui, Agric. Biol. Chem., 40, 2455 (1976).

24) S. Ebashi and F. Ebashi, J. Biochem., 55, 604 (1964). (1964).

25) S. Ebashi and A. Kodama, J. Biochem., 59, 425 (1966).

26) K. Weber and M. Osborne, J. Biol. Chem., 244, 4406 (1969).

27) . Y. Tonomura, "Muscle Proteins, Muscle Contraction and Cation Transport," University of Tokyo Press, Tokyo, 1972, pp. 131, 145, 161.

28) M. Elizinga, J. H. Collins, W. M. Kuehl and R. S. Adelstein, Proc. Natl. Acad. Sci. U.S.A., 70, 2687 (1973).

29) F. Oosawa and M. Kasai, "Subunits in Biological Systems," ed. by S. N. Timasheff and G. D. Fassman, Marcel Dekker Inc., New York, 1971, p. 261.

30) A. Persechini, U. Mrwa and D. J. Hartshorne, 
Biochem. Biophys. Res. Commun., 98, 800 (1981).

31) D. Mornet, R. Bertrand, P. Pantel, E. Audemard and R. Kassab, Biochemistry, 20, 2110 (1981).

32) S. S. Margossian and S. Lowey, Biochemistry, 17, 5431 (1978).

33) H. E. Huxley, J. Mol. Biol., 7, 281 (1963).

34) J. A. Spudich, H. E. Huxley and J. T. Finch, J. Mol. Biol., 72, 619 (1972).

35) K. Maruyama and S. Abe, J. Biochem., 71, 1073 (1972).
36) S. Abe and K. Maruyama, Biochim. Biophys. Acta, 160, 160 (1974).

37) J. Trinick and G. Offer, J. Mol. Biol., 133, 549 (1979).

38) W. Lehman and A. G. Szent-Györgyi, J. Gen. Physiol., 66, 1 (1975).

39) M. C. Schaub, D. J. Hartshorne and S. V. Perry, Biochem. J., 104, 263 (1967).

40) C. S. Cheng, D. D. Hamannan N. B. Webb, J. Food Sci., 44, 1080 (1979). 